\title{
PATTERN ANALYSIS IN NETWORKS OF DIFFUSIVELY COUPLED LUR'E SYSTEMS
}

\author{
Kirill Rogov, Alexander Pogromsky \\ Department of Mechanical Engineering, \\ Eindhoven University of Technology, The Netherlands, \\ k.rogov@tue.nl,a.pogromski@tue.nl \\ Erik Steur \\ Delft Center for Systems and Control, \\ Delft University of Technology, The Netherlands, \\ e.steur@tudelft.nl \\ Wim Michiels \\ Department of Computer Science, \\ Catholic University of Leuven, Belgium, \\ wim.michiels@cs.kuleuven.be \\ Henk Nijmeijer \\ Department of Mechanical Engineering, \\ Eindhoven University of Technology, The Netherlands, \\ h.nijmeijer@tue.nl
}

Received (to be inserted by publisher)

\begin{abstract}
In this paper, a method for pattern analysis in networks of diffusively coupled non-linear systems of Lur'e form is presented. We consider the class of nonlinear systems which are inert or globally asymptotically stable by themselves. Interconnecting such systems into a network via diffusive coupling can result in persistent oscillatory behavior, which may lead to pattern formation in the coupled systems. Some of these patterns may co-exist, which implies multistability of the network dynamics. Multi-stability makes the application of common analysis methods, such as the direct Lyapunov method, highly involved. We develop a numerically efficient method in order to analyze the oscillatory behavior occurring in such networks. It is shown that the oscillations appear via a Hopf bifurcation with the (diffusively) coupling strength as a bifurcation parameter and therefore display sinusoidal-like behavior in the neighbourhood of the bifurcation point. Using the describing function method, we replace nonlinearities with their linear approximations and then analyze the system of linear equations by means of the multivariable harmonic balance method. We show that the multivariable harmonic balance method is able to accurately predict patterns that appear in such a network, even if multiple patterns co-exist.
\end{abstract}

Keywords: Periodic Solutions, Bifurcations, Harmonic Balance, Nonlinear Systems 


\section{Introduction}

In recent years, the study of complex networks consisting of coupled nonlinear dynamical systems has been a significant subject in mathematical biology, control theory, applied physics, and interdisciplinary fields. Its relevance is due to several factors: complex networks are prevalent in the world (e.g. neuroscience, heart cells synchronization, social networks, flocks of birds and other kinds of collective behavior), such networks show a rich dynamic behavior and may induce a large number of different applications (see [Pikovsky et al., 2001], [Strogatz, 2003] and references therein). In particular, different synchronous regimes and pattern formation in coupled systems are subjects of intense study.

Numerous activities in nature exhibit oscillatory behavior and can be described by a collection of coupled nonlinear oscillators. Due to complex dynamics and different types of coupling, various phenomena appear in nonlinear networks. An omnipresent form of interaction is diffusive coupling ([Hale, 1997]), in which the feedback to each (sub)system in the network is a (weighted) sum of differences in each (sub)system's output and those of its neighbours. Networks with diffusive coupling exhibit surprisingly rich phenomena, such as synchronization ([Pogromsky \& Nijmeijer, 2001]), partial synchronization ([Pogromsky et al., 2011]), and formation of oscillatory patterns including standing, travelling and rotating waves (see [Golubitsky et al., 1999] and [Iwasaki, 2008]). Moreover, some of these patterns may co-exist and can be stable for the same set of parameters [Nakao \& Mikhailov, 2010]. These patterns arise from a diffusion-driven instability discovered by [Turing, 1952]. In that research, the author "suggests that a system of chemical substances reacting together and diffusing through a tissue is adequate to account for the main phenomena of morphogenesis". Several concepts related to the chemical basis of morphogenesis (development of patterns), spatial chemical patterns, and what is now called diffusion-driven instability are introduced. In general, the pattern formation phenomenon refers to the generation of complex organization in space and time.

A nonlinear analysis of Turing instability or the diffusion-driven instability phenomenon is described in [Smale, 1976]. In this paper, an example of two systems which are diffusively coupled is studied. Each individual system represents a cell which is inert or dead in the sense that it is globally asymptotically stable. However, when the cells are coupled via diffusion, "the cellular system pulses (or expressed perhaps overdramatically, becomes alive!) in the sense that the concentration of enzymes in each cell will oscillate infinitely". The author poses the question of determining conditions under which the diffusive coupling leads to oscillatory behavior in the network of initially globally asymptotically stable systems. Related results are found in [Yakubovich \& Tomberg, 1989], [Tomberg \& Yakubovich, 2000], and [Pogromsky et al., 1999], and references therein.

Another related result is presented in [Pogromsky et al., 2011]. In that work, the authors present conditions under which a network of systems which are globally asymptotically stable by themselves, being diffusively coupled display different synchronous regimes, such as synchronous and partial synchronous (oscillatory) behavior. These conditions are related to the coupling strength between the agents of the network. When the largest eigenvalue of the coupling matrix exceeds some threshold, the network starts exhibiting oscillatory behavior and this change of dynamics corresponds to a Hopf bifurcation in the network. The Hopf bifurcation changes the dynamics of the network from a stable equilibrium to oscillatory patterns. In this work we want to extend the previously obtained results. The problem of prediction of oscillatory patterns which appear in the (diffusively) coupled Lur'e systems when the Hopf bifurcation occurs is considered in this paper. Another problem arises from the fact that there may exist different oscillatory patterns, which can occur in a network with diffusive coupling, such as an in-phase and an anti-phase synchronization, and some of these patterns can even co-exist, such as clockwise and counterclockwise waves in a ring-like structure. This co-existence implies multistability of the network dynamics. Considering the multistability, the application of common methods for the analysis of network behavior, such as the direct Lyapunov method and the analysis of Poincaré maps, becomes highly non-trivial.

To solve these problems, we propose an extension of the multivariable harmonic balance (MHB) method described in [Iwasaki, 2008] for a neuronal circuits called the central pattern generators (CPGs). The method allows to analyse the behavior of the complex network near the bifurcation point and to determine an oscillatory profile that approximates the output of the studied network without simulating ordinary differential equations (ODEs). Despite these advantages, the MHB method cannot be directly applied to a network consisting of arbitrary systems and in order to achieve the best accuracy of the approximation the coupled systems must be in the neighbourhood of the Hopf bifurcation point. Being inspired by previous results obtained in [Pogromsky et al., 2011] and [Iwasaki, 2008], we developed a new approach in order to analyze oscillatory behavior and predict the patterns with equal ampli- 
tudes formed in the complex networks by means of the multivariable harmonic balance (MHB) method (see [Rogov et al., 2018]). In this work, we extend the results achieved in [Rogov et al., 2018] and present the approach based on the MHB method aiming at pattern analysis with arbitrary amplitudes of oscillations in networks of (diffusively) coupled identical nonlinear systems.

This paper is organized as follows. Preliminary results and the problem statement are given in Section 2. More precisely, in Subsection 2.1, networks of Lur'e systems are introduced. Oscillatory conditions are presented in Subsection 2.2. The Harmonic Balance and the describing function methods are introduced in Subsection 2.3. The problem is formulated in Subsection 2.4. Pattern analysis using the multivariable harmonic balance is described in Section 3. There are four subsections in Section 3. Subsection 3.1 describes the multivariable harmonic balance method presented in [Iwasaki, 2008]. The MHB equation is derived in Subsection 3.2. Subsections 3.3 and 3.4 introduce the equal amplitude case and the general amplitude case, respectively. Numerical results are given in Section 4 and include results for the equal amplitude case and the general amplitude case. In Section 5, the conclusions are provided.

Throughout the paper, the following notations are used. $I_{k}$ and $I_{n}$ denote the identity matrices of the size $k \times k$ and $n \times n$, respectively. ${ }^{\top}$ stands for the transposition. $i$ stands for the imaginary unit. $C^{1}$ stands for a set of continuously differentiable functions.

\section{Preliminaries and problem statement}

In this section we state the problem, describe the class of systems which are considered and impose related assumptions. Subsection 2.1 introduces dynamical systems and the type of coupling that form networks whose dynamics are analyzed in this work. In Subsection 2.2, conditions that guarantee the occurrence of oscillatory patterns are provided. Tools being used for the analysis of network behavior are introduced in Subsection 2.3. The formulation of the problem is given in Subsection 2.4.

\subsection{Networks of Lur'e systems}

In this paper, a method to analyze the behavior of networks consisting of identical nonlinear dynamical systems is presented. To do so, we consider a network which describes a collection of identical (diffusively) coupled (sub)systems. It is assumed that the network cannot be decomposed into two or more uncoupled networks. To turn the statements above into a mathematical description, we consider a network of $k$ identical systems described by:

$$
\begin{aligned}
\dot{x}_{j} & =A x_{j}+B u_{j}, \\
u_{j} & =u_{c j}-\psi\left(z_{j}\right), \\
z_{j} & =Z x_{j}, \\
y_{j} & =C x_{j},
\end{aligned}
$$

where $j=1, \ldots, k$ stands for number of the node, $x_{j}(t) \in \mathbb{R}^{n}$ is the state of a $j$-th (sub)system, $u_{j}(t) \in \mathbb{R}^{1}$ is the combined input of a $j$-th (sub)system which consists of two components: $\psi(\cdot)$ which is a continuously differentiable scalar nonlinear sector-bounded function and $u_{c j} \in \mathbb{R}^{1}$ which is the input receiving data from the coupling of multiple (sub)systems, $y_{j}(t) \in \mathbb{R}^{1}$ is the output of the $j$-th system used for the coupling, $z_{j} \in \mathbb{R}^{1}$ is the argument of the nonlinearity of a $j$-th (sub)system and $A, B, C, Z$ are constant matrices of appropriate dimension. Taking $u_{c j}=0$, we have $k$ isolated single input single output systems given in Lur'e form. An arbitrary number of identical systems can be mutually connected via the inputs $u_{j}$ and the outputs $y_{j}$ into a network. An example of such a network is shown in the Figure 1.

The subsystems are interconnected through linear diffusive output coupling

$$
u_{c j}=\gamma\left(-\gamma_{j 1}\left(y_{j}-y_{1}\right)-\gamma_{j 2}\left(y_{j}-y_{2}\right)-\ldots-\gamma_{j k}\left(y_{j}-y_{k}\right)\right),
$$

where $\gamma$ is the positive overall gain and also the bifurcation parameter, $\gamma_{j l}$ are non-negative constants and $\gamma_{j l}=\gamma_{l j}$. Moreover, $\gamma_{j l}>0$ if and only if the $j$-th and $l$-th nodes are connected. We define the coupling matrix $\Gamma$ of size $k \times k$ 


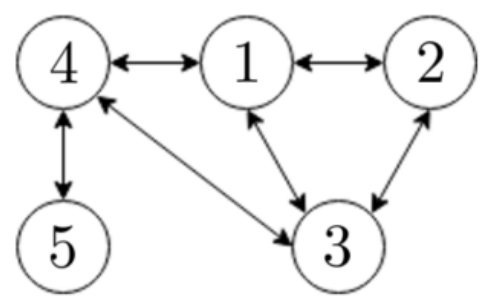

Fig. 1. An example of the network consisting of dynamical systems.

with all row sums zero and $\Gamma=\Gamma^{\top}$ and thus all eigenvalues are non-negative [Gershgorin, 1931], i.e.

$$
\Gamma=\gamma\left(\begin{array}{cccc}
\sum_{l=2}^{k} \gamma_{1 j} & -\gamma_{12} & \ldots & -\gamma_{1 k} \\
-\gamma_{21} & \sum_{l=1, l \neq 2}^{k} \gamma_{2 j} & \ldots & -\gamma_{2 k} \\
\vdots & \vdots & \ddots & \vdots \\
-\gamma_{k 1} & -\gamma_{k 2} & \ldots & \sum_{l=1}^{k-1} \gamma_{k j}
\end{array}\right) .
$$

Using the Laplace transformation, the time-invariant linear part of the network (1) is rewritten into the transfer function representation, leading to the following description of the closed loop system

$$
\begin{aligned}
y_{j} & =W_{y}(s) u_{j}, \\
z_{j} & =W_{z}(s) u_{j}, \\
u_{j} & =-\sum_{l=1}^{k} \gamma_{j l} y_{j}-v_{j}, \\
v_{j} & =\psi\left(z_{j}\right),
\end{aligned}
$$

with $W_{y}(s)$ and $W_{z}(s)$ defined as

$$
\begin{aligned}
& W_{y}(s)=C(s I-A)^{-1} B, \\
& W_{z}(s)=Z(s I-A)^{-1} B .
\end{aligned}
$$

\subsection{Conditions for oscillatory behavior}

The goal of this subsection is to present conditions that guarantee that the network (1)-(2) exhibits oscillatory behavior. The system (1)-(2) is called Y-oscillatory if system's solutions are ultimately bounded and there exist a $j \in\{1, \ldots, k\}$ such that for almost all initial conditions $x(0) \in \mathbb{R}^{k n} \lim _{t \rightarrow+\infty} y_{j}(t)$ does not exist [Yakubovich, 1973].

Networks of the form (1)-(2) were considered in [Pogromsky et al., 2011] and it was shown that individual dynamics of the (sub)systems of the network satisfies some conditions for network synchronization.

Assumption 1. The individual dynamics of system (1) and transfer functions (5) and (6) satisfy the following conditions:

i) Matrix $A$ is Hurwitz, so there is a positive definite matrix $P=P^{\top}$, so that $A^{\top} P+P A=-I$;

ii) Matrix $Z$ satisfies $Z^{\top}=P B$ with matrix $P$ as in i);

iii) $\psi(\cdot)$ is an odd, strictly increasing $C^{1}$-function with the following property $\forall L>0 \exists \sigma>0$ such that $\forall z>\sigma$ $\psi(z)>L z$

iv) Transfer function $W_{y}(s)$ is non-degenerate, has an even number of zeros with positive real part and relative degree one, and $W_{y}(0)>0$;

v) Transfer function $W_{z}(s)$ is such that $W_{z}(0)>0$.

The following theorem presented in [Pogromsky et al., 2011] provides a condition under which a network that satisfies Assumption 1, exhibits oscillatory behavior. 
Theorem 1. Consider a network of diffusively coupled systems of the form (4) that satisfy Assumption 1 with a symmetric coupling matrix $\Gamma$ as in (3).

There is a number $\bar{\lambda}>0$ such that if the largest non-zero eigenvalue $\lambda_{\max }$ of $\Gamma$ exceeds $\bar{\lambda}$ then the network is Y-oscillatory.

The proof of the theorem captures three points:

i) The origin is the unique equilibrium point of the closed loop system;

ii) The system is uniformly ultimately bounded (see [Khalil, 1996]);

iii) The origin is hyperbolically unstable if $\lambda_{\max }>\bar{\lambda}$, where $\lambda_{\max }$ is the largest eigenvalue of $\Gamma$.

The dynamics of a network has at least two different modes (a globally asymptotically stable mode and a Yoscillatory mode) depending on the value of parameter $\gamma$. In this work, we consider the case when a Hopf bifurcation occurs in a network at the point, where the largest eigenvalue of matrix $\Gamma$ parametrized by bifurcation parameter $\gamma$, $\lambda_{\max }=\bar{\lambda}$. The Hopf bifurcation changes the dynamics from the globally asymptotically stable equilibria to the oscillatory behavior. The bifurcation parameter is represented as the overall gain of coupling strength between the nodes $\gamma$. The Hopf bifurcation point can be easily computed for any network consisting of nonlinear dynamical systems of the form (4). The Hopf bifurcation of an equilibrium is characterized by one bifurcation condition which is the presence of a purely imaginary pair of eigenvalues of the Jacobian matrix evaluated at this equilibrium [Kuznetsov, 1998]. For some networks the largest eigenvalue $\lambda_{\max }$ of the coupling matrix $\Gamma$ has algebraic and geometric multiplicity larger than one or, in other words, an equivariant Hopf bifurcation occurs [Balanov, 1997]. Assuming that $\psi^{\prime}(0)=0$, the Hopf bifurcation (equivariant Hopf bifurcation) occurs when matrix $A-B C \bar{\lambda}$ has a pair of purely imaginary eigenvalues with algebraic and geometric multiplicity one (larger than one) and the other eigenvalues have negative real part. We also compute bifurcation frequency $\omega_{\text {bif }}$ which will be required later on.

According to normal form theory (see [Han \& Yu, 2012] and references therein), the oscillations are sinusoidal-like near the bifurcation point. It justifies using the describing function method to replace the nonlinearity by its linear approximation and then to analyze the system of linear equations by means of the harmonic balance method.

\subsection{Harmonic Balance Method and Describing function}

The harmonic balance method provides an analytical approximation of periodic solution(s) of an ODE and is applicable to nonlinear systems. The considered (sub)system (1) is nonlinear and the nonlinearity $\psi(\cdot)$ can be approximated by its describing function. Suppose a (sub)system of the Y-oscillatory network has a periodic solution $z_{j}(t)$. Then, by Fourier series expansion, we obtain

$$
z_{j}(t)=\sum_{m=0}^{\infty} a_{j m} \sin (\omega m t)+b_{j m} \cos (\omega m t)
$$

for real vectors $a_{j m}$ and $b_{j m}$ and frequency $\omega$. In the neighbourhood of the bifurcation point the solution is approximated by its first harmonic

$$
z_{j}(t) \cong \alpha_{j} \sin \left(\omega t+\phi_{0}+\phi_{j}\right),
$$

where $\alpha_{j}$ is the amplitude, $\omega$ is the frequency, $\phi_{0}$ is the initial phase and $\phi_{j}$ is the phase for the $j$-th subsystem of the network. For an autonomous system we can take $\phi_{0}$ to be equal to 0 without loss of generality. In order to approximate the nonlinearity $\psi(\cdot)$ by its describing function $k$, we first compute the describing function which stands for the gain and then replace the nonlinearity with the linear gain as follows:

$$
\psi\left(z_{j}\right) \cong k\left(\alpha_{j}\right) z_{j}
$$

where

$$
k\left(\alpha_{j}\right):=\frac{2}{\pi \alpha_{j}} \int_{0}^{\pi} \psi\left(\alpha_{j} \sin \theta\right) \sin \theta \mathrm{d} \theta .
$$

The describing function $k\left(\alpha_{j}\right)$ stands for the gain of the nonlinearity $\psi(\cdot)$ when the input of the nonlinearity is a sinusoidal signal of amplitude $\alpha_{j}$ ([Khalil, 1996]). The harmonic balance method for networks of CPGs (Central Pattern Generators) [see Section 3.1 for details] called the Multivariable Harmonic Balance (MHB), was described in [Iwasaki, 2008]. 


\subsection{Problem formulation}

The goal of this work is to present a method which is able to analyze oscillatory patterns appearing in networks of diffusively coupled identical nonlinear systems. Networks of the form (1)-(2), whose (sub)system's individual dynamics satisfy Assumption 1, exhibit oscillatory behavior if the coupling between network agents is strong enough. These patterns can be characterized by a set of parameters (oscillatory profiles), such as amplitudes $\alpha_{j}$, phases $\phi_{j}$ and frequency $\omega$. Predicting a pattern implies that its oscillatory profile $\left(\alpha_{j}, \phi_{j}, \omega\right)$ can be computed by MHB analysis and oscillations can be reconstructed from this profile using (7). Some of these patterns can co-exist, which implies the multistability of the network dynamics. In Section 2.2, it is argued that a Hopf bifurcation (equivariant Hopf bifurcation) is the origin of the oscillations, which justifies the use of an approach based on the harmonic balance method. The harmonic balance method helps to avoid a classical analysis, e.g., by means of the direct Lyapunov method. Moreover, the direct Lyapunov method is difficult to apply when we deal with multistability. In this work, we present the Multivariable Harmonic Balance method aiming at pattern prediction in Y-oscillatory networks consisting of Lur'e systems with a diffusive coupling. In other words, we generalize the method presented in [Iwasaki, 2008]to networks of (diffusively) coupled Lur'e systems. The goal of the method is to derive an oscillatory profile of patterns consisting of amplitudes $\alpha_{j}$, phases $\phi_{j}$ and frequency $\omega$ and then to reconstruct periodic solutions using (7).

\section{Pattern Prediction by means of Multivariable Harmonic Balance}

In this section, we review the multivariable harmonic balance (MHB) method which we extend in order to analyze the behavior of networks of the form (4) when pattern formation occurs. The MHB method approximates the behavior of a network consisting of nonlinear systems and determines an oscillatory profile (frequency $\omega$, amplitudes $\alpha_{j}$, phases $\phi_{j}$ ) as a solution to an eigenvalue problem. In Subsection 3.1, the Multivariable Harmonic Balance described in [Iwasaki, 2008] is introduced. In Subsection 3.2, we show how the MHB method is applied to the network (1)-(2). There are two cases to discuss namely the equal amplitude case and the general amplitude case which are described in Subsections 3.3 and 3.4, respectively.

\subsection{Multivariable Harmonic Balance}

In [Iwasaki, 2008] one considers a network of identical interconnected (sub)systems described by a dynamical mapping from input $u_{j}$ to output $z_{j}$ :

$$
z_{j}(s)=f(s) u_{j}(s), \quad u_{j}(s)=\sum_{l=1}^{k} \mu_{j l}(s) v_{l}, \quad v_{j}=\psi\left(z_{j}\right),
$$

where $f(s)$ is a rational transfer function of variable $s$, which represents the linear time-invariant part of each (sub)system, $\psi\left(z_{j}\right)$ is a continuously differentiable odd static nonlinear function, $z_{j}(s)$ is the $j$-th (sub)system output and $\mu_{j k}(s)$ is a transfer function representing the connection from $l$-th system to $j$-th system. In vector form, the dynamics can be written as

$$
z=F(s) u, \quad u=M(s) v, \quad v=\Psi(z),
$$

where $\Psi(z):=\operatorname{col}\left[\psi\left(z_{1}\right), \ldots, \psi\left(z_{k}\right)\right], z:=\operatorname{col}\left[z_{1}, \ldots, z_{k}\right], F(s):=f(s) I_{k}$ and $M(s)$ is the transfer matrix of the coupling whose $(j, k)$ entry is $\mu_{j k}(s)$. Using equations (7)-(9), the dynamic equations are approximated by the MHB equation

$$
\left(\mathscr{M}(s) \mathscr{K}(\alpha)-I_{k}\right) q=0, q_{j}:=\alpha_{j} e^{i \phi_{j}}, q \in \mathbb{C}^{k},
$$

where $\mathscr{M}(s)=F(s) M(s), \mathscr{K}(\alpha):=k\left(\alpha_{j}\right) I_{k}$ is a diagonal matrix and $q$ is a solution to the eigenvalue problem. The MHB equation characterizes the oscillatory profiles for (10) as follows. In order to obtain approximations of oscillatory solutions, we substitute a sample solution of the form $q_{j}=\alpha_{j} \exp \left(i \phi_{j}\right)$. All three components $(\alpha, \phi, \omega)$ of the pattern are computed and the oscillatory profile is reconstructed using (7).

In general, the MHB equation is nonlinear in $\alpha$ and might have multiple solutions and it is necessary to determine the stable oscillations. In order to examine stability of such oscillatory solution, we use an approximate method from [Glad \& Ljung, 2000] commonly accepted in the engineering literature. Based on (8), we consider the linear systems 
obtained by replacing the non-linearity $\Psi$ with constant gain $\mathscr{K}(\alpha)$ as follows.

$$
L_{K}:=\left\{\begin{array}{l}
z=\mathscr{M}(s) v, \\
v=\mathscr{K}(\alpha) z .
\end{array}\right.
$$

The block scheme representation of the linear system $L_{K}$ is illustrated in Figure 2. From the perspective of the describing function method, the linear system $L_{K}$ contains some information on the network behavior when its oscillations are sinusoidal-like with a set of amplitudes $\alpha$. According to the approximate method from [Glad \& Ljung, 2000], the oscillation with certain $\omega, \alpha, \phi$ is stable if the linear approximation $L_{K}$ is marginally stable, which is equivalent to the boundedness of all solutions of 12 .

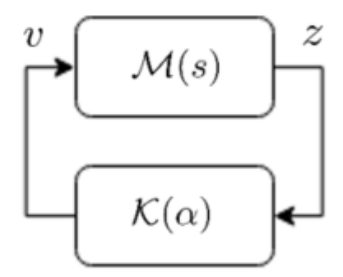

Fig. 2. Linear system $L_{K}$.

From the above, the key ideas of the MHB analysis are summarised as follows:

- The oscillatory profile can be obtained by solving equation (11) for frequency $\omega$ and eigenvector $z$ containing encoded amplitudes and phases.

- The predicted oscillatory pattern is stable if the corresponding linear system $L_{K}$ given in (12) with $K:=\mathscr{K}(\alpha)$ is marginally stable with a pair (pairs) characteristic roots at $s= \pm i \omega$ on the imaginary axis and with the rest characteristic roots located in the open left half plane.

\subsection{Deriving the MHB equation}

It is shown that the MHB method turns the problem of determining oscillatory profiles into an eigenvalue problem. System (4) does not belong to the class of systems considered in [Iwasaki, 2008], so the previously described MHB method cannot be directly applied. In [Iwasaki, 2008], it was assumed that the transfer matrix of coupling $M(s)$ introduced in (10) is constant, which does not hold for our problem. In order to apply the MHB analysis, a loop transformation is performed. Figure 3 displays how the loop transformation reorders the structure of the network. The left and right blocks describe the networks (4) and (10), respectively.
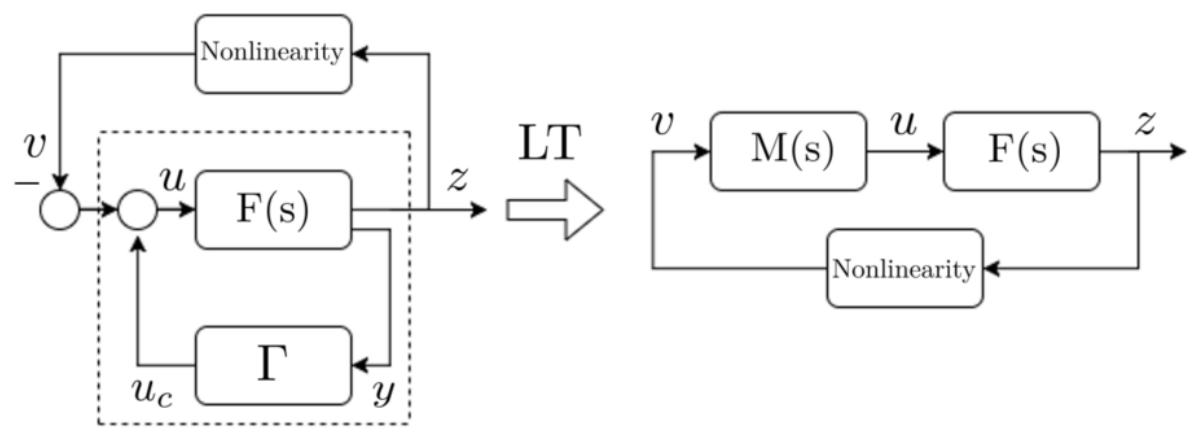

Fig. 3. Loop Transformation reorders the structure of the network in order to apply the MHB method. 
The following manipulations are performed to turn system (4) into the form of (10). Recall System (4) and rewrite the input $u$ in vector form as

$$
\begin{aligned}
& u=-W_{y}(s) \Gamma u-v \\
& W_{y}(s)\left[\frac{1}{W_{y}(s)} I_{k}+\Gamma\right] u=-v,
\end{aligned}
$$

with matrix $\Gamma$ defined in (3). Defining the resolvent $R\left(W_{y}^{-1}(s)\right)=\left[W_{y}^{-1}(s) I+\Gamma\right]^{-1}$, we obtain

$$
u=-W_{y}^{-1}(s) R\left(W_{y}^{-1}(s)\right) v .
$$

The network now takes the form of (10) with

$$
\begin{aligned}
& F(s)=W_{z}(s) I_{k}, \\
& M(s)=-W_{y}^{-1}(s)\left[W_{y}^{-1}(s) I_{k}+\Gamma\right]^{-1} .
\end{aligned}
$$

Combining the second equation from (10) with (13) and replacing $s$ by $i \omega$, we arrive at the following equation

$$
\mathscr{M}(i \omega)=-\frac{F(i \omega)}{W_{y}(i \omega)}\left(\frac{1}{W_{y}(i \omega)} I_{k}+\Gamma\right)^{-1} .
$$

Define the diagonal matrix

$$
H(i \omega):=\frac{F(i \omega)}{W_{y}(i \omega)}=\left(\frac{W_{z}(i \omega)}{W_{y}(i \omega)}\right) I_{k}
$$

and substitute it in the previous equation in order to obtain

$$
\mathscr{M}(i \omega)=-H(i \omega)\left(\frac{1}{W_{y}(i \omega)} I_{k}+\Gamma\right)^{-1} .
$$

Subsequently, we rewrite the MHB equation (11) as follows

$$
\mathscr{M}(i \omega)\left(\mathscr{K}(\alpha)-\mathscr{M}^{-1}(i \omega)\right) q=0,
$$

where $\mathscr{M}^{-1}(i \omega)=-\left(\left(1 / W_{y}(i \omega)\right) I_{k}+\Gamma\right) H(i \omega)^{-1}$ and expand it in order to obtain

$$
\mathscr{M}^{-1}(i \omega)\left(\frac{W_{z}(i \omega)}{W_{y}(i \omega)}\right)\left[\mathscr{K}(\alpha)\left(\frac{W_{z}(i \omega)}{W_{y}(i \omega)}\right)+\frac{1}{W_{y}(i \omega)} I_{k}+\Gamma\right] q=0 .
$$

Since $\mathscr{M}^{-1}(i \omega)\left(W_{z}(i \omega) / W_{y}(i \omega)\right) \neq 0$ for $\omega>0$, we obtain

$$
J(\alpha, \omega) q:=\left[\mathscr{K}(\alpha)\left(\frac{W_{z}(i \omega)}{W_{y}(i \omega)}\right)+\frac{1}{W_{y}(i \omega)} I_{k}+\Gamma\right] q=0, \quad q_{j}:=\alpha_{j} e^{i \phi_{j}}
$$

which is the multivariable harmonic balance equation for the networks of identical Lur'e systems. We focus on determining patterns that appear in such networks. We consider both the equal amplitude case with $\alpha_{j}=\alpha_{e} \forall j$, $\alpha_{e} \in \mathbb{R}^{+}$, and the general amplitude case with possibly (all) different $\alpha_{j} \in \mathbb{R}^{+}, j=1, \ldots, k$.

\subsection{Equal amplitude case}

In this subsection we review the equal amplitude case to gain some insight into the method. This special case is derived for networks exhibiting oscillations with equal amplitudes $\alpha_{j}=\alpha_{e} \forall j$. Consider ring-like networks with three and four nodes (Fig.4). Each (sub)system of these networks is a dynamical system that satisfies Assumption 1 and whose dynamics is presented in Section 4. The network topology is represented by a symmetric unweighted graph with the overall coupling gain. The nodes interconnect with neighbours via diffusive coupling. According to this, considered networks are regular. Networks are referred to as regular if each node has exactly the same number of links [Chen, 1997]. Regularity favours the occurrence of stable oscillations with equal amplitudes. Since we look for $\alpha_{j}=\alpha_{e} \forall j$, the analysis of the network behavior can be substantially simplified using the structural properties of the network. In this subsection, it is shown how the oscillatory profile is determined by the MHB method for the equal amplitude case. 

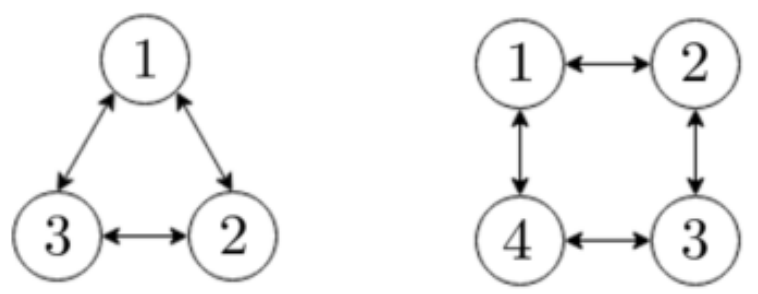

Fig. 4. Ring-like networks of diffusively coupled systems displaying oscillations with equal amplitudes

Fig. 5 reflects the oscillatory patterns that can be observed in the networks shown in Fig.4. Oscillatory profiles are obtained by the direct simulation of ordinary differential equations (ODEs) of networks (1). For presentation purposes, components of $z_{j}(t)$ are shifted as follows $z_{j}(t)+(k+1-j)$. The oscillatory pattern has equal amplitudes with a phase shift of 120 degrees for the three-node configuration which is a rotating wave. Partial synchronization is observed for the four-node configuration. There are two synchronized groups of nodes which are in antiphase with each other.
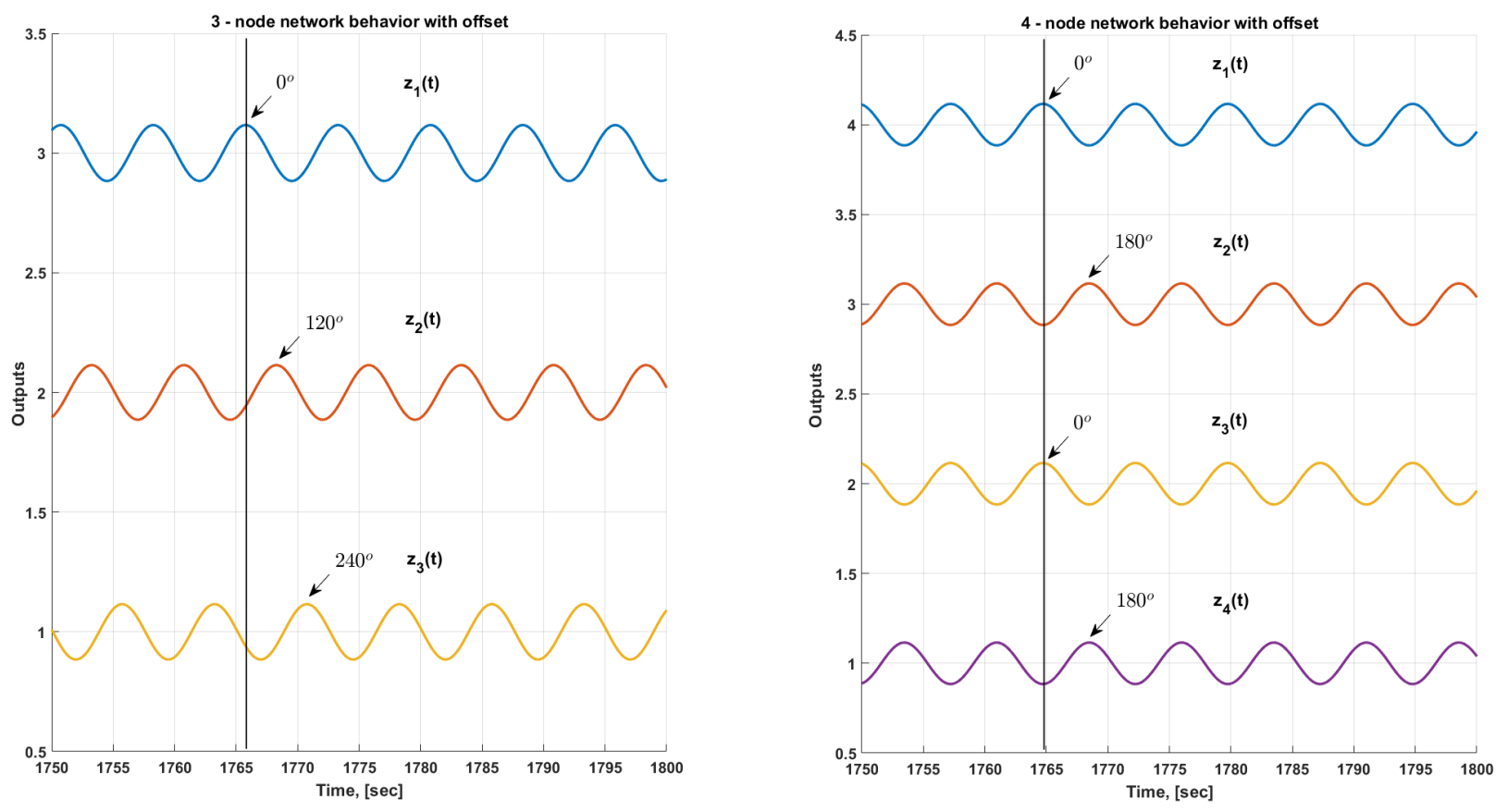

Fig. 5. Oscillatory patterns with equal amplitudes in ring-like networks (left fig.: 3-node network, right fig.: 4-node network). Network dynamics is given in Section 4.

Since we look for equal amplitudes $\alpha_{j}=\alpha_{e} \forall j$, the describing function matrix is now defined as $\mathscr{K}(\alpha)=k\left(\alpha_{e}\right) I_{k}$ and equation (14) reduces to

$$
\left[\frac{k\left(\alpha_{e}\right) W_{z}(i \omega)+1}{W_{y}(i \omega)} I_{k}+\Gamma\right] q=0, \quad q_{j}:=\alpha_{e} e^{i \phi_{j}}
$$

where $q$ is the solution to the eigenvalue problem. One can see that equation (15) has several solutions. The trivial solution $z=0$ corresponds to the equilibrium and is not point of interest. Therefore, the following equation is solved

$$
\operatorname{det}\left[\frac{k\left(\alpha_{e}\right) W_{z}(i \omega)+1}{W_{y}(i \omega)} I_{k}+\Gamma\right]=0
$$


After diagonalizing the coupling matrix $\Gamma$ via a similarity transformation, which is possible due to $\Gamma=\Gamma^{\top}$, and using the property of the determinant product, we arrive at

$$
\frac{k\left(\alpha_{e}\right) W_{z}(i \omega)+1}{W_{y}(i \omega)}+\lambda_{j}=0
$$

where $\lambda_{j}$ is an eigenvalue of the coupling matrix $\Gamma$.

The described procedure implies that different oscillatory patterns may appear based on each eigenpair (an eigenvalue and its corresponding eigenvector) of the matrix $\left[\frac{k\left(\alpha_{e}\right) W_{z}(i \omega)+1}{W_{y}(i \omega)} I_{k}+\Gamma\right]$. Nonetheless, from Theorem 1 , it is known that if $\lambda_{\max }$ exceeds some threshold the network is Y-oscillatory. It was mentioned above that an oscillation is expected to be stable if the linear approximation (12) is marginally stable. Since $\Gamma=\Gamma^{\top}$, the eigenvalues located on the imaginary axis, have linearly independent eigenvectors which are eigenvectors of the coupling matrix $\Gamma$. The eigenvalue with the largest real part $\lambda_{\max }$ of the coupling matrix $\Gamma$ parametrized by bifurcation parameter $\gamma$ characterizes the Hopf bifurcation (equivariant Hopf bifurcation) point in the network. We now consider equation (16) for $\lambda_{\max }$

$$
\frac{k\left(\alpha_{e}\right) W_{z}(i \omega)+1}{W_{y}(i \omega)}+\lambda_{\max }=0
$$

Substituting (5) and (6) into (17), we obtain

$$
\begin{aligned}
k\left(\alpha_{e}\right) Z\left(i \omega I_{k}-A\right)^{-1} B+1+\lambda_{\max } C\left(i \omega I_{n}-A\right)^{-1} B & =0, \\
1+\left(k\left(\alpha_{e}\right) Z+\lambda_{\max } C\right)\left(i \omega I_{n}-A\right)^{-1} B & =0 .
\end{aligned}
$$

Since the isolated system is exponentially stable, $\operatorname{det}\left(i \omega I_{n}-A\right) \neq 0$ for all $\omega$, we have

$$
\left(i \omega I_{k}-A\right)\left[1+\left(k\left(\alpha_{e}\right) Z+\lambda_{\max } C\right)\left(i \omega I_{n}-A\right)^{-1} B\right]=0 .
$$

Using Schur's Lemma (see [Dummit \& Foote, 1999]), we arrive at

$$
\operatorname{det}\left[i \omega I_{n}-A+B\left(k\left(\alpha_{e}\right) Z+\lambda_{\max } C\right)\right]=0 .
$$

Thus the matrix $A-B\left(k\left(\alpha_{e}\right) Z+\lambda_{\max } C\right)$ has a pair of pure imaginary eigenvalues with algebraic and geometric multiplicity one for Hopf bifurcation (larger than one for equivariant Hopf bifurcation) for $\pm i \omega$, where $\omega$ stands for frequency of the oscillations and the other eigenvalues are in the open left-half complex plane

$$
\operatorname{Re}\left(\lambda_{1,2}\left(A-B\left(k\left(\alpha_{e}\right) Z+\lambda_{\max } C\right)\right)\right)=0 .
$$

In other words, $k\left(\alpha_{e}\right)$ and $\omega$ can be derived from the Hopf bifurcation condition. Now two components of the oscillatory profile are obtained: $\alpha_{e}$ using (9) and $\omega$. The phases $\phi_{j}$ are encoded in the eigenvector that corresponds to the largest eigenvalue $\lambda_{\max }$ of the coupling matrix $\Gamma$.

Substituting $\left(k\left(\alpha_{e}\right) W_{z}(i \omega)+1\right) / W_{y}(i \omega)=-\lambda_{\max }$ in (15), we obtain

$$
\left[\Gamma-\lambda_{\text {max }} I_{k}\right] q=0 .
$$

Since we look for equal amplitudes, we need such eigenvector $q$ with the entries represented as $q_{j}=\alpha_{j} \exp \left(i \phi_{j}\right)$ that all of $q_{j}$ have the same modulus $\alpha_{e}$. In order to determine the phases from the eigenvector the following equation is used

$$
\phi_{j}=\operatorname{angle}\left(q_{j}\right) .
$$

Having all components of the oscillatory profile computed, the approximation of the oscillatory pattern of the given network using the equation (7) can be constructed.

It was mentioned above that multiple oscillatory patterns occurring in such networks may co-exist. Co-existence implies that there simultaneously exist at least two stable solutions in ring-like structured networks, such as clockwise and counter-clockwise rotating waves. This happens when the largest eigenvalue $\lambda_{\max }$ of the coupling matrix $\Gamma$ has algebraic and geometric multiplicity two which implies the existence of two linearly independent eigenvectors corresponding to this eigenvalue or, in other words, an equivariant Hopf bifurcation occurs [Balanov, 1997]. The phases of clockwise and counter-clockwise rotating waves are encoded in these eigenvectors and be determined using (20). 


\subsection{General amplitude case}

In this subsection a method for estimating the profile of oscillations with arbitrary amplitudes is presented. Networks exhibiting oscillatory patterns with different amplitudes can be easily obtained by attaching additional nodes to the networks presented in Fig.4 as shown in Fig.6. These networks consist of identical dynamical systems that satisfy Assumption 1 and whose individual dynamics is given in Section 4. As in the equal amplitude case, we consider networks whose topology is described by a symmetric unweighted graph with the overall coupling gain. Unlike the equal amplitude case, these networks are not regular.
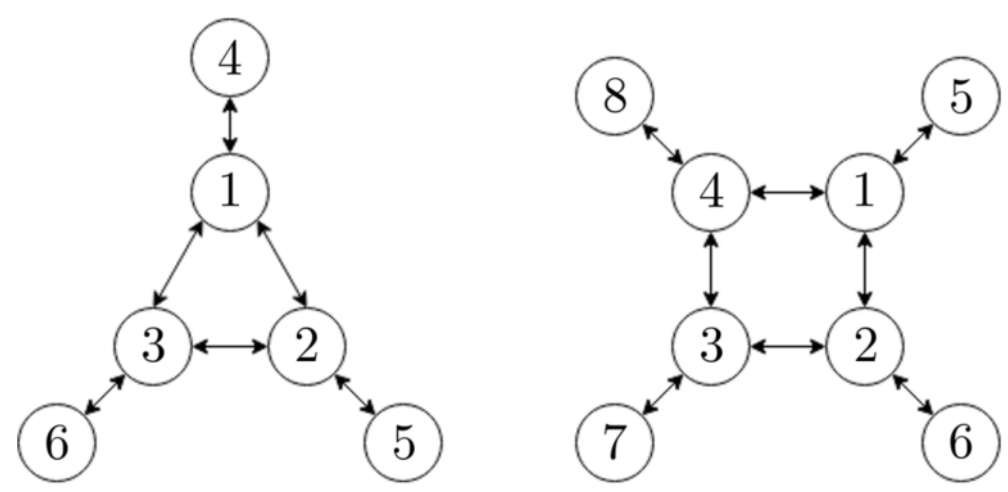

Fig. 6. Networks of identical diffusively coupled systems displaying oscillations with unequal amplitudes.

Such networks are perfect candidates to illustrate and study the general amplitude case due to their structure and behavior. Fig. 7 reflects the oscillatory patterns that can be observed in the networks shown in Fig.6. Results are obtained by the direct simulation. For presentation purposes, components of $z_{j}(t)$ are shifted as follows $z_{j}(t)+(k+$ $1-j)$. Two groups of nodes with equal amplitudes are observed. The first group corresponds to the core ring which is the subnetwork of nodes with more than one neighbour. Core rings of the networks from Fig.6 belong to the first group and the attached nodes belong to the second group.
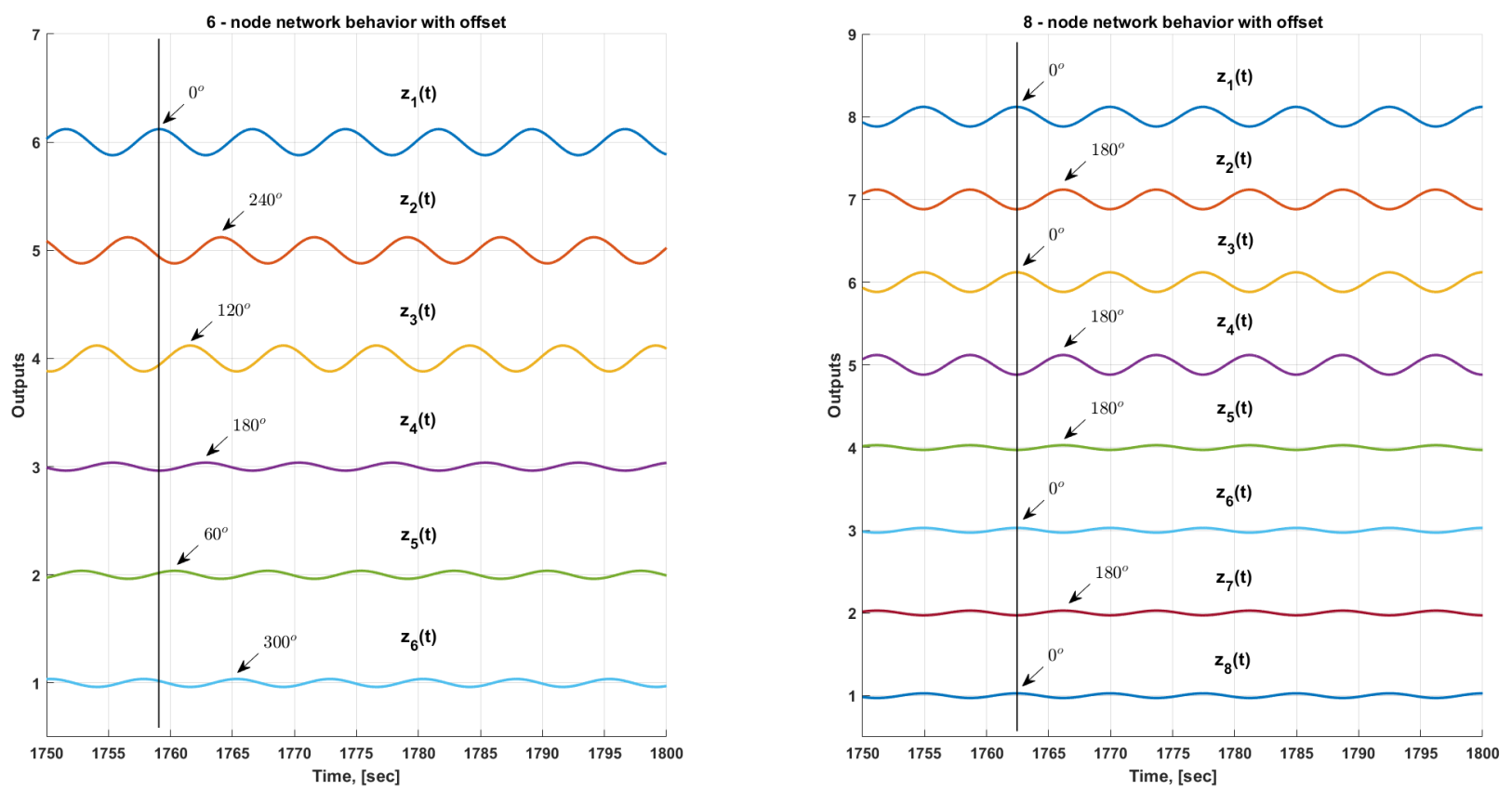

Fig. 7. Oscillatory patterns with unequal amplitudes (left fig.: 6-node network, right fig.: 8-node network). Network dynamics is given in Section 4. 
Recall equation (14),

$$
J(\alpha, \omega) q:=\left[\mathscr{K}(\alpha)\left(\frac{W_{z}(i \omega)}{W_{y}(i \omega)}\right)+\frac{1}{W_{y}(i \omega)} I_{k}+\Gamma\right] q=0 .
$$

To solve the equation above, numerical methods are employed. Since the trivial solution $q=0$ is not a point of interest, we look for a set of phases $\phi_{j}$, amplitudes $\alpha_{j}$ and frequency $\omega$ which solves the nonlinear equation (14). Define the objective function to minimize as

$$
F(g):=Z^{\prime} Z+p^{\top} p
$$

where

$$
g:=[\phi ; \alpha ; \omega] \in \mathbb{R}^{2 k+1}, \quad Z:=J(\alpha, \omega) q \in \mathbb{C}^{k}, \quad p:=\alpha-|q| \in \mathbb{R}^{k} .
$$

The vector $g$ contains unknown phases, amplitudes and frequency, the component $p$ represents the second equation of (14). To simplify the problem the phase of the first node is fixed at $\phi_{1}=0$ and boundaries are chosen as: $\phi_{j} \in[0,2 \pi] \forall j \neq 1, \alpha_{j} \in[0, \beta] \forall j, \omega \in\left[0,2 \omega_{b i f}\right]$, where $\beta$ is a heuristically chosen number. A smart choice of $\beta$ allows substantial decrease in convergence time.

Solutions to nonlinear equation (14) are found by minimizing the squared residual norm of the objective function (21). The local solver fmincon included in MATLAB environment [MATLAB, 2017] is used. The solver uses the interior-point algorithm [Byrd et al., 2000] in order to find a minimum of constrained nonlinear multivariable function in defined feasible region. Since equations (14) are nonlinear and multiple solutions exist, the solutions heavily depend on the initial conditions. Multiple values of the initial conditions are generated by the MultiStart algorithm [Ugray et al., 2007].

A big advantage of the method is that start points for the minimization procedure are not needed to be specified. A set of random points is used as initial conditions. The key point in the procedure is to choose the correct boundaries for the start points and the unknown variables.

The procedure has the following steps:

(1) Define the objective function to minimize;

(2) Define the constraints for start points and unknowns;

(3) Set start points for the solver (random or custom);

(4) Run the solver.

Moreover, the MultiStart algorithm can deal with multi-stability. Stable clockwise and counter-clockwise wavelike solutions can be simultaneously found.

\section{Numerical examples}

In this section, it is shown how the multi-variable harmonic balance method is applicable to networks consisting of dynamical systems of Lur'e form whose structures given in the Figures 4 and 6 . These networks are perfect candidates to demonstrate the performance of the method. The networks exhibit different oscillatory patterns, such as patterns with equal and unequal amplitudes, patterns with a simple phase shift (180 degrees) and with non-trivial phase shift (e.g. 120 degrees), and co-existing clockwise and counter-clockwise wave-like patterns. The individual dynamics of the nodes, as in [Pogromsky et al., 2011], is given in (1) with

$$
\begin{gathered}
A=\left(\begin{array}{rrr}
1 & -1 & 1 \\
1 & 0 & 0 \\
-4 & 2 & -3
\end{array}\right) \\
B=\left(\begin{array}{lll}
0 & 0 & 1
\end{array}\right)^{\top}, C=\left(\begin{array}{lll}
0 & 0 & 1
\end{array}\right), Z=B^{\top} P,
\end{gathered}
$$

where $P$ is the solution to the Lyapunov equation

$$
A^{\top} P+P A=-I_{3}
$$

The nonlinear function $\psi(\cdot)$ is given as follows

$$
\psi\left(z_{j}\right)=z_{j}^{3}
$$


and computing the describing function by (9), we obtain

$$
k\left(\alpha_{j}\right)=\frac{3}{4} \alpha_{j}^{2} .
$$

The presented setting satisfies the conditions from Assumption 1. Since numerical methods are employed, it implies that a computational error exists which is given as residual of Eq.(14). In order to show, that the MHB method accurately predicts oscillatory patterns, the reconstructed oscillations and the output of a direct simulation are compared. The direct simulation results are obtained using a nonstiff differential equation solver of medium order ode 45 included in MATLAB environment [Dormand \& Prince, 1980]. In order to measure the accuracy of the method we provide residual norm of the MHB equation and compare amplitudes and frequency with the ones that are extracted from ODE simulation as follows.

$$
\begin{aligned}
& \Delta \alpha=100\left\|\alpha-\alpha_{s}\right\| /\left\|\alpha_{s}\right\| ; \\
& \Delta \omega=100\left(\omega-\omega_{s}\right) / \omega_{s},
\end{aligned}
$$

where $\alpha_{s}$ and $\omega_{s}$ are taken from the simulation. In the following two subsections, we will present results of the analytical approach for the equal amplitude case and the numerical approach for the general amplitude case.

\subsection{Results for the equal amplitude case}

In this subsection, we show that it is possible predict patterns occurring in regular networks with certain symmetry and that oscillatory patterns possess equal amplitudes. An example of such networks is given in the Figure 4. It is shown how to refine an oscillatory profile of the given network by means of the analytical approach presented above. This approach is applicable only for networks exhibiting patterns with equal amplitudes.

The coupling topology of the networks are given by matrices $\Gamma_{3}$ and $\Gamma_{4}$ where a subscript denotes the number of nodes in the network

$$
\Gamma_{3}=\gamma_{3}\left(\begin{array}{rrr}
2 & -1 & -1 \\
-1 & 2 & -1 \\
-1 & -1 & 2
\end{array}\right), \quad \Gamma_{4}=\gamma_{4}\left(\begin{array}{rrrr}
2 & -1 & 0 & -1 \\
-1 & 2 & -1 & 0 \\
0 & -1 & 2 & -1 \\
-1 & 0 & -1 & 2
\end{array}\right)
$$

where $\gamma_{3,4}$ are bifurcation parameters. In the Section 2, it is shown that the Hopf bifurcation (equivariant Hopf bifurcation) occurs in the network when the largest eigenvalue $\lambda_{\max }$ with algebraic and geometric multiplicity one (larger than one) of the coupling matrix parametrized by bifurcation parameter $\gamma$ exceeds some threshold $\bar{\lambda}$. There exist positive numbers $\bar{\lambda}_{3}, \bar{\lambda}_{4}$ so that if $\bar{\lambda}_{3}<\gamma_{3}$ and $\bar{\lambda}_{4}<\gamma_{4}$ for the three node and four node networks, respectively, the systems are Y-oscillatory. Parameters $\bar{\lambda}_{3}, \bar{\lambda}_{4}$ can be calculated from the Hopf bifurcation (equivariant Hopf bifurcation) condition $A-B C \bar{\lambda}$ described in Section 2.2 and for the given networks these are: $\bar{\lambda}_{3}=0.43426$ and $\bar{\lambda}_{4}=0.32569$.

For the three node network, two stable wave-like oscillatory patterns (clockwise and counter-clockwise waves) are found and the oscillation parameters are successfully determined. The accuracy of the MHB method and the derived oscillatory profiles for the coupling strength $\gamma_{3}=1.01$ are presented in Table 1 .

Table 1. Oscillatory profiles of the network represented by matrix $\Gamma_{3}$. Multi-stability.

\begin{tabular}{ccccccccc}
\hline Wave & Residual & $\Delta \alpha$ in $\%$ & $\Delta \omega$ in $\%$ & $\omega$ & $\alpha_{e}$ & $\phi_{1}$ & $\phi_{2}$ & $\phi_{3}$ \\
\hline Clockwise & $9.9256 \mathrm{e}-07$ & 0.5781 & 0.0371 & 0.8365 & 0.1296 & 0 & 240 & 120 \\
Counterclockwise & $9.9256 \mathrm{e}-07$ & 0.5781 & 0.0371 & 0.8365 & 0.1296 & 0 & 120 & 240 \\
\hline
\end{tabular}

For the four node network, one wave-like solution is predicted. The predicted solution corresponds to a partial synchronization regime. We can observe synchronous behavior between two pairs of nodes, namely between the 1 st and the 3rd, and between the 2 nd and the 4 th. Moreover, these pairs are in anti-phase synchronization with respect to 
each other. The accuracy of the MHB method and the predicted oscillatory profile for the coupling strength $\gamma_{4}=1.01$ are provided in Table 2.

Table 2. Oscillatory profile and comparison of the MHB method and ODE simulation of the network represented by matrix $\Gamma_{4}$.

\begin{tabular}{ccccccccc}
\hline Residual & $\Delta \alpha$ in $\%$ & $\Delta \omega$ in $\%$ & $\omega$ & $\alpha_{e}$ & $\phi_{1}$ & $\phi_{2}$ & $\phi_{3}$ & $\phi_{4}$ \\
\hline $1.0171 \mathrm{e}-06$ & 1.0793 & 0.0521 & 0.8362 & 0.1159 & 0 & 180 & 0 & 180 \\
\hline
\end{tabular}

In the case of the four node network, the MHB method predicts the result which was previously obtained by means of the direct Lyapunov method in [Pogromsky et al., 2011]. For the three node case, the analysis by means of the direct Lyapunov method is cumbersome due to the co-existence of two stable solutions.
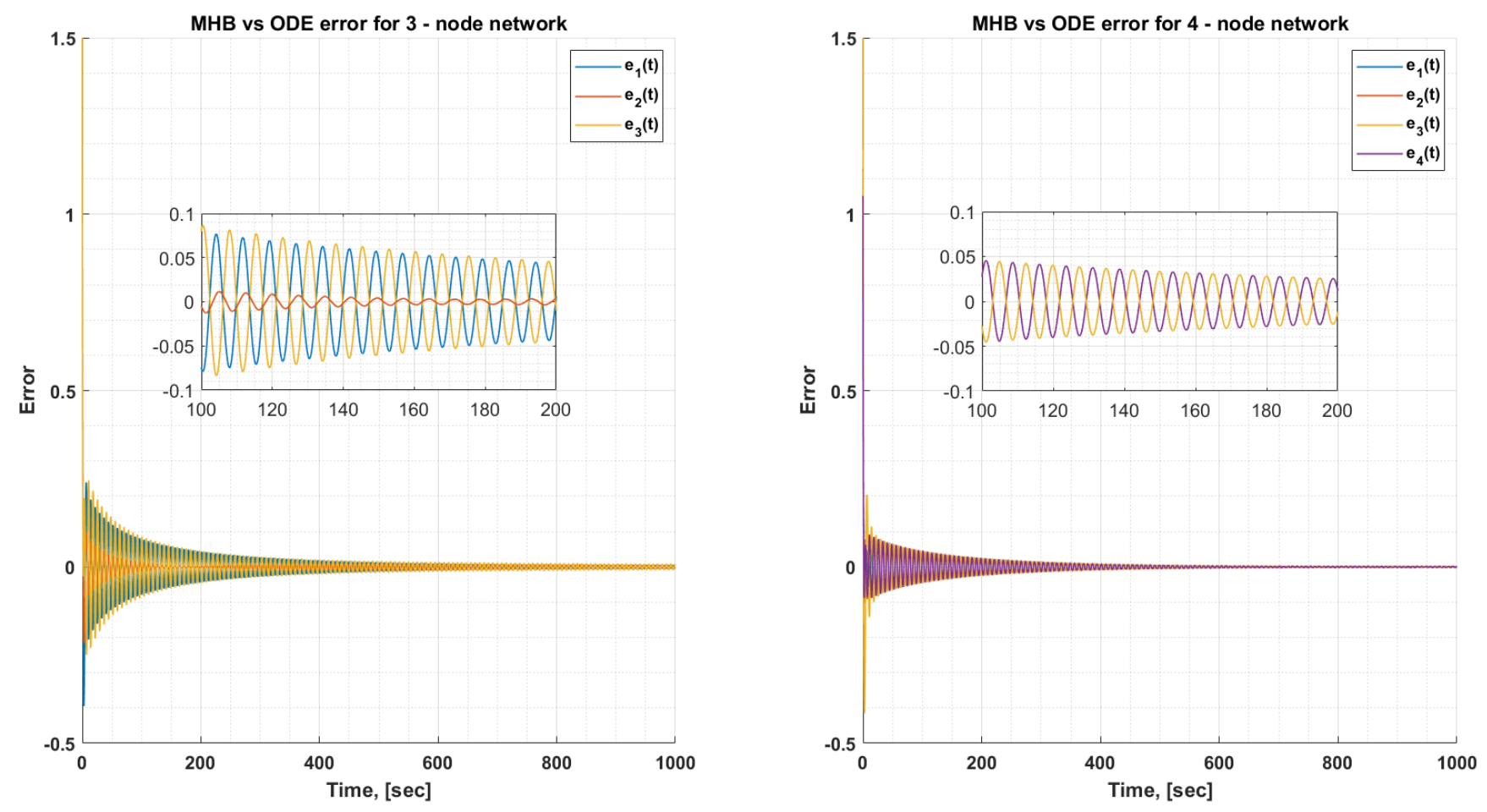

Fig. 8. Error between the MHB method and ODE simulations for three- and four-node networks for $\gamma_{3,4}=1.01 \bar{\lambda}_{3,4}$. Inner plots represent zoomed-in sections for the presentation purposes.

Figure 8 shows that in the initial period of time the oscillations computed by the simulation and the output of the MHB method do not match because of transients. When the transients have sufficiently decayed the outputs are almost identical which implies that the MHB method accurately describes the patterns occurring in the networks. The vertical axis stands for the mismatch between outputs of the simulation and the MHB method and the mismatch is computed as $e(t)=z(t)-q(t)$. The horizontal axis stands for time. For some initial conditions, oscillations computed by the MHB method have different initial phases comparing to the simulation ones. In order to compensate this difference, an initial phase offset $\phi_{0}$ can be applied to the MHB output vector as shown in (7). 


\subsection{Results for the general amplitude case}

In this subsection, the results obtained by means of the optimization tools are presented. The numerical approach seeks for the set of the phases, amplitudes, and frequency that solves equation (14). This approach is applicable to networks exhibiting patterns with not necessarily equal amplitudes. Despite this, it is preferred to use the analytical approach for the equal amplitude case in order to save computation time. In the equal amplitude case the structure of the network can be exploited in such a way that one ends up with an eigenvalue problem of size $n$ instead of $n k$. Here we present the analysis of the networks which are given in Figure 6 and whose structure is described by matrices $\Gamma_{6}$ and $\Gamma_{8}$ where a subscript denotes the number of nodes in the network:

$$
\Gamma_{6}=\gamma_{6}\left(\begin{array}{rrrrrr}
3 & -1 & -1 & -1 & 0 & 0 \\
-1 & 3 & -1 & 0 & -1 & 0 \\
-1 & -1 & 3 & 0 & 0 & -1 \\
-1 & 0 & 0 & 1 & 0 & 0 \\
0 & -1 & 0 & 0 & 1 & 0 \\
0 & 0 & -1 & 0 & 0 & 1
\end{array}\right), \quad \Gamma_{8}=\gamma_{8}\left(\begin{array}{rrrrrrrr}
3-1 & 0 & -1 & -1 & 0 & 0 & 0 \\
-1 & 3 & -1 & 0 & 0 & -1 & 0 & 0 \\
0 & -1 & 3 & -1 & 0 & 0 & -1 & 0 \\
-1 & 0 & -1 & 3 & 0 & 0 & 0 & -1 \\
-1 & 0 & 0 & 0 & 1 & 0 & 0 & 0 \\
0 & -1 & 0 & 0 & 0 & 1 & 0 & 0 \\
0 & 0 & -1 & 0 & 0 & 0 & 1 & 0 \\
0 & 0 & 0 & -1 & 0 & 0 & 0 & 1
\end{array}\right),
$$

where $\gamma_{6,8}$ are bifurcation parameters. The Hopf bifurcation (equivariant Hopf bifurcation) occurs in these networks when the largest eigenvalue of matrix $\Gamma_{6,8}$ exceeds $\bar{\lambda}_{6}=0.30278$ and $\bar{\lambda}_{8}=0.24881$ for the six node network and the eight node network, respectively.

We use the MultiStart optimization framework in order to predict patterns that appear in the given networks. The objective function is given in (21), without loss of generality, we set the first phase $\phi_{1}=0$ in order to simplify the problem, and boundaries are chosen to be $\phi_{j} \in[0,2 \pi] \forall j \neq 1, \alpha_{j} \in[0, \beta] \forall j, \omega \in\left[0,2 \omega_{b i f}\right]$, where $\omega_{\text {bif } 6,8}=0.8350$ and $\beta=0.2$ is heuristically chosen. In order to show the accuracy of the MHB method for the general amplitude case, the error analysis is performed, that is why the three values mentioned above are used (i.e. Residual norm of the MHB equation, $\Delta \alpha$, and $\Delta \omega$ ). First, we present the results for the network of six nodes and the coupling strength parameter $\gamma_{6}=1.01$. The procedure starts from ten random points within the chosen boundaries and two stable solutions are found that represent the clockwise and counter-clockwise waves. Existence of these waves implies the multistability of the network dynamics. The determined oscillatory patterns are given in Table 3 and the accuracy of the method is given in Table 4.

Table 3. Oscillatory profiles of the network represented by matrix $\Gamma_{6}$. Multi-stability.

\begin{tabular}{cccccccccccccc}
\hline Wave & $\omega$ & $\alpha_{1}$ & $\alpha_{2}$ & $\alpha_{3}$ & $\alpha_{4}$ & $\alpha_{5}$ & $\alpha_{6}$ & $\phi_{1}$ & $\phi_{2}$ & $\phi_{3}$ & $\phi_{4}$ & $\phi_{5}$ & $\phi_{6}$ \\
\hline CW & 0.8364 & 0.1226 & 0.1242 & 0.1242 & 0.0375 & 0.0380 & 0.0380 & 0 & 240 & 120 & 180 & 60 & 300 \\
CCW & 0.8364 & 0.1226 & 0.1242 & 0.1242 & 0.0375 & 0.0380 & 0.0380 & 0 & 120 & 240 & 180 & 300 & 60 \\
\hline
\end{tabular}

Table 4. Comparison of the MHB method and ODE simulation applied to the network represented by matrix $\Gamma_{6}$. Multi-stability.

\begin{tabular}{cccc}
\hline Wave & Residual & $\Delta \alpha$ in $\%$ & $\Delta \omega$ in $\%$ \\
\hline CW & 0.00016 & 2.5387 & 0.0499 \\
CCW & 0.00016 & 2.5080 & 0.0230 \\
\hline
\end{tabular}

One can see that the multivariable harmonic balance method accurately predicts the oscillatory patterns which appear in such networks, even for co-existing patterns. 
The objective function (21) has the argument $g \in \mathbb{R}^{2 k+1}$. It is not possible to visualize the function of of this dimension in 3D and that is why we provide cross-sections of the objective function. We change two out of the $2 k+1$ variables within some range and fix the remaining variables to be constant. Figures 9 and 10 show cross-sections that we choose in order to display that the computed solutions correspond to local minima. Figure 10 shows that there exist local minima for the clockwise and counter-clockwise wave-like solutions which implies multi-stability of the network.

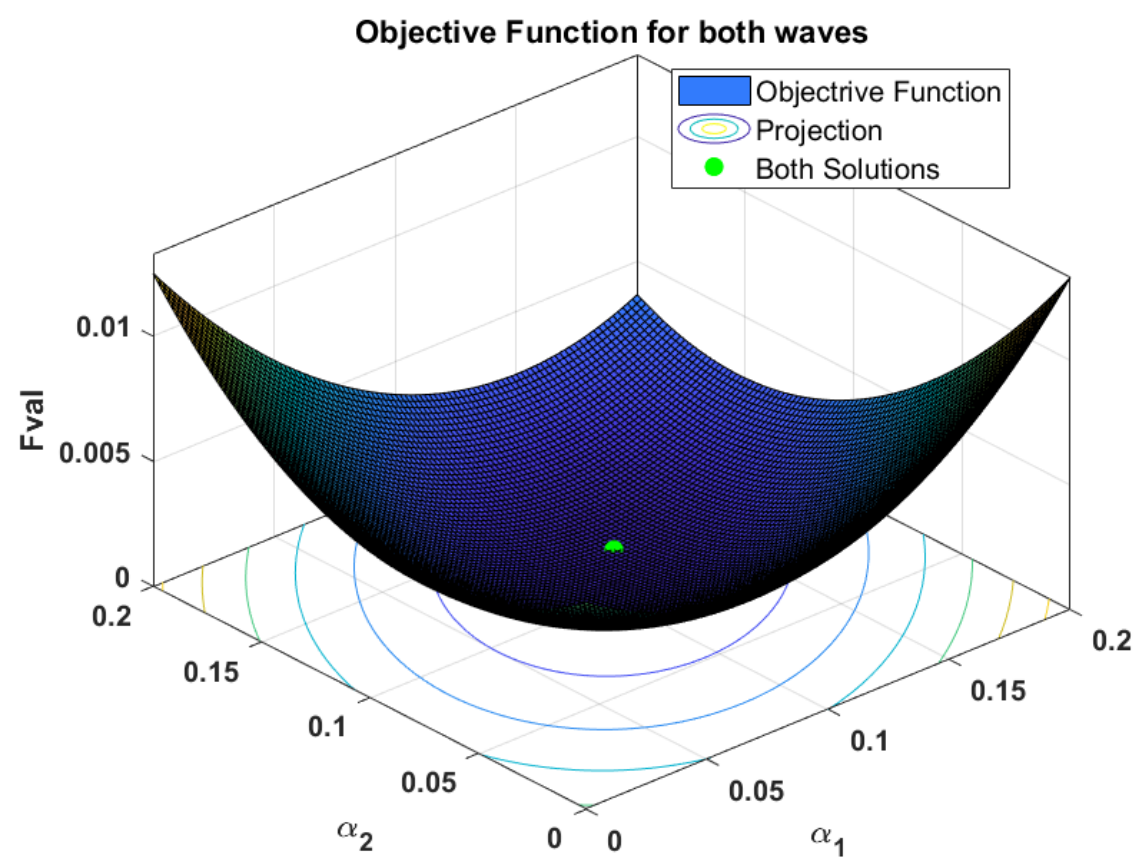

Fig. 9. Objective Function cross-section of $\alpha_{1}$ and $\alpha_{2}$ for clockwise and counterclockwise waves. Since both wave-like solutions has equal amplitudes $\alpha_{1}$ and $\alpha_{2}$, they are shown as a single point.

We have the same setting (ten random points within the chosen boundaries) for the eight node network whose structure is represented by coupling matrix $\Gamma_{8}$. Multi-stability is not observed in this network. The coupling strength parameter is chosen to be $\gamma_{8}=1.01$. The determined oscillatory pattern is provided in Table 5 and the accuracy of the method is given in Table 6 .

Table 5. Oscillatory profile of the network represented by matrix $\Gamma_{8}$.

\begin{tabular}{cccccccccccccccccccccc}
\hline$\omega$ & $\alpha_{1}$ & $\alpha_{2}$ & $\alpha_{3}$ & $\alpha_{4}$ & $\alpha_{5}$ & $\alpha_{6}$ & $\alpha_{7}$ & $\alpha_{8}$ & $\phi_{1}$ & $\phi_{2}$ & $\phi_{3}$ & $\phi_{4}$ & $\phi_{5}$ & $\phi_{6}$ & $\phi_{7}$ & $\phi_{8}$ \\
\hline 0.8363 & 0.1209 & 0.1209 & 0.1209 & 0.1209 & 0.0289 & 0.0289 & 0.0289 & 0.0289 & 0 & 180 & 0 & 180 & 180 & 0 & 180 & 0 \\
\hline
\end{tabular}

In a network of an even number of nodes in a "core" ring, we observe partial synchronization and simple phase shift of 180 degrees. As it was mentioned above, the MHB method approximates a non-linearity by a linear gain. Theoretically, the accuracy of the approximation becomes better as the network moves closer to a bifurcation point. We show how the accuracy changes when we increase the coupling strength. The example of eight nodes is used to illustrate this dependence and the results are presented in Table 7 and Figure 11. We run the method for the eight node network but for different coupling strength parameter $\gamma_{8}$. We performed six runs increasing $\gamma_{8}$ from 1.01 till 1.5 . 

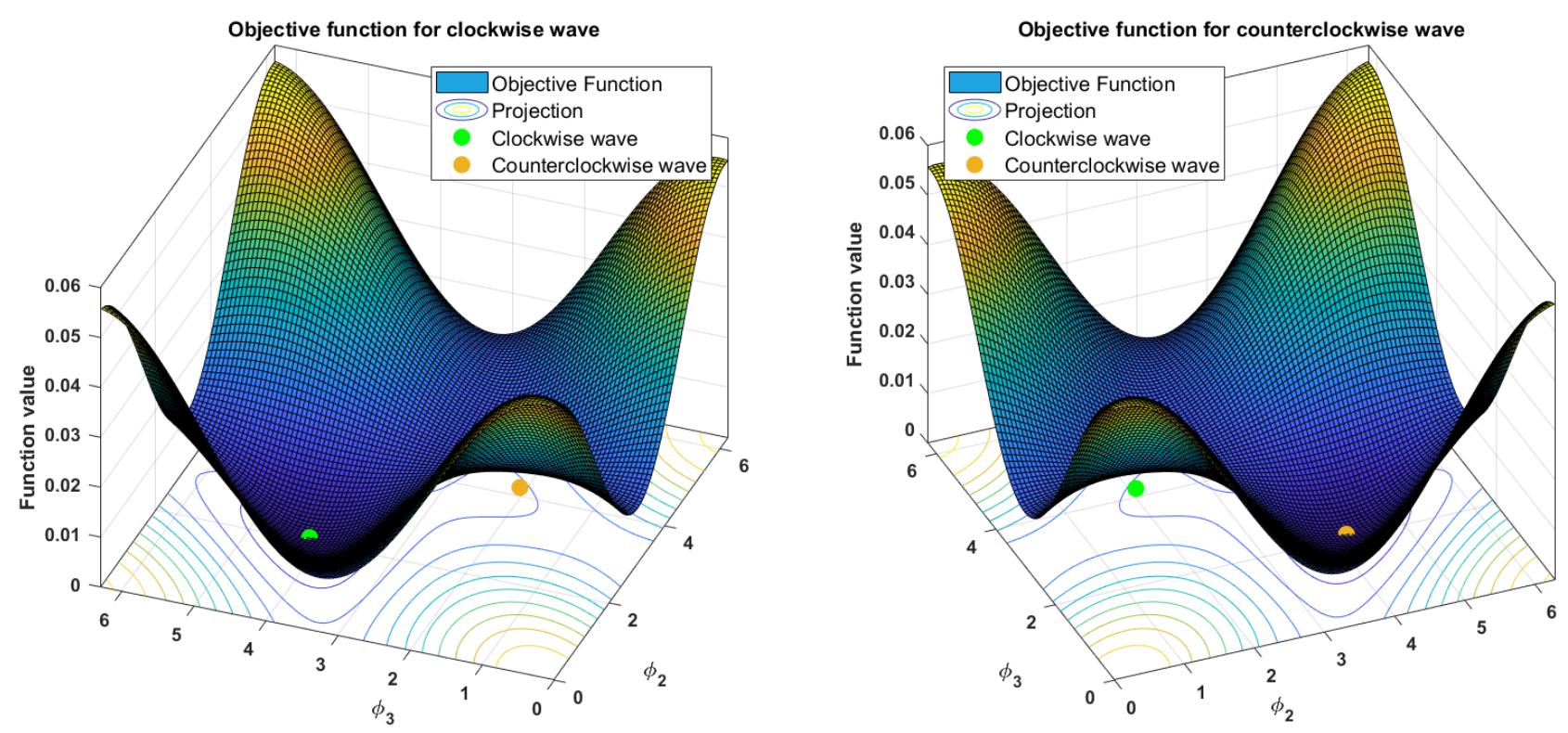

Fig. 10. Objective Function cross-section of $\phi_{2}$ and $\phi_{3}$ for both solutions.

Table 6. Comparison of the MHB method and ODE simulation applied to the network represented by matrix $\Gamma_{8}$.

\begin{tabular}{ccc}
\hline Residual & $\Delta \alpha$ in $\%$ & $\Delta \omega$ in $\%$ \\
\hline 0.00056 & 2.4267 & 0.0367 \\
\hline
\end{tabular}

Table 7. Comparison of the MHB method and ODE simulation for 8-node network vs. coupling strength.

\begin{tabular}{ccccccc}
\hline Run & 1 & 2 & 3 & 4 & 5 & 6 \\
\hline$\gamma$ & 1.01 & 1.1 & 1.2 & 1.3 & 1.4 & 1.5 \\
Residual & 0.0005 & 0.0003 & 0.0003 & 0.0003 & 0.0003 & 0.0568 \\
$\Delta \omega$ in \% & 0.0127 & 0.0242 & 0.0798 & 0.1135 & 0.2266 & 0.3262 \\
$\Delta \alpha$ in \% & 0.7264 & 0.7905 & 0.8440 & 0.9247 & 1.1635 & 1.2844 \\
\hline
\end{tabular}

The numbers in Table 7 and Figure 11 illustrate that the accuracy changes as it was predicted and the mismatch increases when the system goes away from the Hopf bifurcation (equivariant Hopf bifurcation) point. This data shows that the MHB method can accurately predict oscillatory patterns even if the system is "far" from the bifurcation point.

\section{Conclusion}

This work addresses the problem of predicting patterned oscillations of arbitrary amplitudes in networks of identical nonlinear dynamical systems of Lur'e form. The patterns are characterized by a set of parameters consisting of frequency $\omega$, amplitudes $\alpha_{j}$ and phases $\phi_{j}$. In this work, we consider the case when the Hopf bifurcation (equivariant Hopf bifurcation) is the origin of oscillations and the oscillations are sinusoidal-line in the neighbourhood of the 

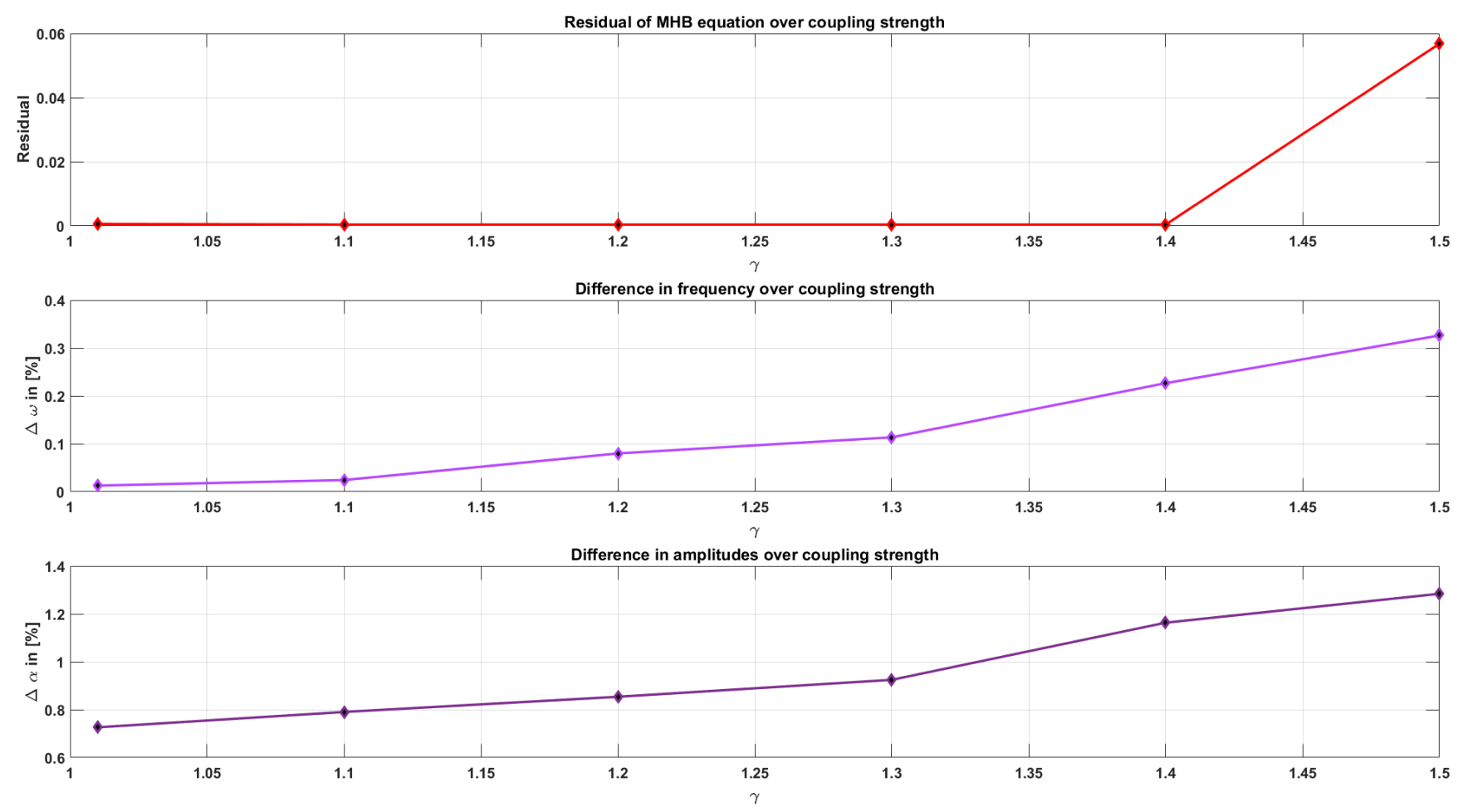

Fig. 11. Comparison of the MHB method and ODE simulation for network represented by $\Gamma_{8}$ for different coupling strength.

bifurcation point. According to this the nonlinearity can be approximated by its describing function and the network behavior can be analyzed by means of the harmonic balance method. Moreover, these patterns may co-exist which implies multistability. Multistability makes the usage of common tools, such as the direct Lyapunov method, highly involved. The multivariable harmonic balance (MHB) method for coupled systems of specific class was presented in [Iwasaki, 2008]. The method is able to determine the oscillatory profile of the patterns that occur in the network of specific structure and deals with multistability. The MHB method is not directly applicable to the networks of interest and in this work, we present a modification of the MHB method for the networks consisting of the Lur'e systems coupled via diffusion. The multivariable harmonic balance equation for networks of Lur'e systems is derived and it is shown that the method can accurately predict patterns that are formed in the networks of our interest. For this, two cases are considered the equal amplitude case and the general amplitude case. The equal amplitude case can be easily solved using the structural properties of the network. The general amplitude case requires numerical analysis. A numerical optimization routine has been developed which determines optimal solutions to the MHB equation and therewith predicts all possible stable patterns of oscillations.

It is shown that the phenomenon of pattern formation occurring in networks of (diffusively) coupled Lur'e systems can be analysed by means of the multivariable harmonic balance method and the further research goal is to apply the MHB method to a collection of interconnected neuronal systems (e.g. FitzHughNagumo model) with delays in coupling.

\section{Acknowledgements}

The author received funding from the European Union's Horizon 2020 research and innovation programme under the Marie Skłodowska-Curie grant agreement No 675080.

\section{References}

Balanov, Z. [1997] "Equivariant Hopf Theorem," Nonlinear Analysis: Theory, Methods and Applications 30, 34633474. 
Byrd, R. H., Gilbert, J. C. \& Nocedal, J. [2000] "A trust region method based on interior point techniques for nonlinear programming," Mathematical Programming 89, 149-185.

Chen, W. K. [1997] Graph Theory and Its Engineering Applications (WORLD SCIENTIFIC).

Dormand, J. R. \& Prince, P. J. [1980] “A family of embedded Runge-Kutta formulae,” J. Comp. Appl. Math. 6, 1926.

Dummit, D. S. \& Foote, R. M. [1999] Abstract Algebra (2nd ed.) (New York: Wiley), ISBN 0-471-36857-1.

Gershgorin, S. [1931] "Uber die abgrenzung der eigenwerte einer matrix," Bulletin de l'Académie des Sciences de l'URSS. Classe des sciences mathématiques et na 6, 749-754.

Glad, T. \& Ljung, L. [2000] Control theory - multivariable and nonlinear methods (Taylor and Francis).

Golubitsky, M., Stewart, I., Buono, P.-1. \& Collins, J. J. [1999] "Symmetry in locomotor central pattern generators and animal gaits," Nature 401, 693-695.

Hale, J. [1997] "Diffusive coupling, dissipation, and synchronization," Journal of Dynamics and Differential Equations 9, 1-52.

Han, M. \& Yu, P. [2012] "Hopf Bifurcation and Normal Form Computation," Normal Forms, Melnikov Functions and Bifurcations of Limit Cycles (Springer London), ISBN 978-1-4471-2917-2, pp. 7-58.

Iwasaki, T. [2008] "Multivariable harmonic balance for central pattern generators," Automatica 44, 3061-3069.

Khalil, H. K. [1996] Nonlinear Systems (Prentice-Hall, New Jersey).

Kuznetsov, Y. A. [1998] Elements of Applied Bifurcation Theory (2Nd Ed.) (Springer-Verlag, Berlin, Heidelberg), ISBN 0-387-98382-1.

MATLAB [2017] MATLAB Optimization Toolbox (R2017a) (The MathWorks Inc., Natick, Massachusetts).

Nakao, H. \& Mikhailov, A. S. [2010] "Turing patterns in network-organized activator-inhibitor systems," Nature Physics 6, 544-550.

Pikovsky, A., Rosenblum, M. \& Kurths, J. [2001] Synchronization, Cambridge Nonlinear Science Series (Cambridge University Press), ISBN 9780511755743.

Pogromsky, A., Glad, T. \& Nijmeijer, H. [1999] “on Diffusion Driven Oscillations in Coupled Dynamical Systems," International Journal of Bifurcation and Chaos 09, 629-644.

Pogromsky, A., Kuznetsov, N. \& Leonov, G. [2011] "Pattern generation in diffusive networks: How do those brainless centipedes walk?" Proceedings of the IEEE Conference on Decision and Control , 7849-7854.

Pogromsky, A. \& Nijmeijer, H. [2001] "Cooperative oscillatory behavior of mutually coupled dynamical systems," IEEE Transactions on Circuits and Systems I: Fundamental Theory and Applications 48, 152-162.

Rogov, K., Pogromsky, A., Steur, E., Michiels, W. \& Nijmeijer, H. [2018] "Pattern prediction in networks of diffusively coupled nonlinear systems," IFAC-PapersOnLine 51, 62 - 67, 5th IFAC Conference on Analysis and Control of Chaotic Systems CHAOS 2018.

Smale, S. [1976] "A Mathematical Model of Two Cells Via Turing's Equation," The Hopf Bifurcation and Its Applications (Springer New York), ISBN 978-1-4612-6374-6, pp. 354-367.

Strogatz, S. [2003] Sync: The Emerging Science of Spontaneous Order (Hyperion Press), ISBN 0786868449.

Tomberg, E. \& Yakubovich, V. [2000] “On one problem of Smale," Siberian Mathematical Journal 4, 771-774.

Turing, A. [1952] "The chemical basis of morphogenesis," Philosophical Transactions of the Royal Society of London. Series B, Biological Sciences 237, 37-72.

Ugray, Z., Lasdon, L., Plummer, J., Glover, F., Kelly, J. \& Mart, R. [2007] "Scatter search and local nlp solvers: A multistart framework for global optimization,” INFORMS Journal on Computing 19, 328-340.

Yakubovich, V. [1973] "Frequency conditions for auto-oscillations in nonlinear systems with one stationary nonlinearity," Siberian Mathematical Journal 14, 768-788.

Yakubovich, V. \& Tomberg, E. [1989] "Conditions for auto-oscillations in nonlinear systems," Siberian Mathematical Journal 30, 641-653. 